\title{
Some problems about geometric lattice
}

\author{
$\operatorname{Lin} \mathrm{Si}^{1}$ \\ College of Science, Beijing Forestry University \\ 35 Qinghua East Road, Beijing 100083,P.R.China
}

\begin{abstract}
In this paper, the survey about some results of the convex lattice set are given and the invariance of projection problem of convex lattice set is also obtained. And combining a famous result in the graph theory, several conjectures about the convex lattice set are presented.
\end{abstract}

\section{Introduction}

A convex body is a compact, convex subset with nonempty interior in the $\boldsymbol{n}$-dimensional Euclidean space $\boldsymbol{R}^{n}$. Aleksandrov's projection theorem[2], states that if the $(\boldsymbol{n}-1)-$ dimensional volume $\boldsymbol{v}_{\boldsymbol{n}-1}$ of projections of two origin-symmetric convex bodies $\boldsymbol{K}_{1}$ and $\boldsymbol{K}_{2}$ are equal in every direction, then $\boldsymbol{K}_{1}$ and $\boldsymbol{K}_{2}$ are translates of one another.

Let $\boldsymbol{S}^{n-1}$ denote the unit sphere and $\boldsymbol{u}^{\perp}$ denote the $(\boldsymbol{n}-1)-$ dimensional subspace orthogonal to $\boldsymbol{u}$. The usual orthogonal projection of $\boldsymbol{K}$ on $\boldsymbol{u}^{\perp}$ is $\boldsymbol{K} \mid \boldsymbol{u}^{\perp}$. Then the Aleksandrov's projection theorem can be formularized as the following.

Theorem 1 (Aleksandrov) Let $\boldsymbol{K}_{1}$ and $\boldsymbol{K}_{2}$ be two origin-symmetric convex bodies and $\boldsymbol{v}_{n-1}\left(K_{1} \mid u^{\perp}\right)=v_{n-1}\left(K_{2} \mid u^{\perp}\right)$ for all $u \in S^{n-1}$

Then $\boldsymbol{K}_{1}$ and $\boldsymbol{K}_{2}$ coincide up to a translation.

According to the development of the theory of convex body, results about the discrete analog of convex body are very few. In this note, a survey of the projection problem of convex lattice set is given. And several conjectures about the convex lattice set are suggested.

\section{Discrete version of the Aleksandrov problem}

Let $\operatorname{conv}(\boldsymbol{K})$ denote the convex hull of a set $\boldsymbol{K}$. A convex lattice set $\boldsymbol{F}$ is a finite subset of the $\boldsymbol{n}-$ dimensional integer lattice $\boldsymbol{Z}^{n}$ such that $\boldsymbol{F}=\boldsymbol{\operatorname { c o n v }}(\boldsymbol{F}) \cap \boldsymbol{Z}^{\boldsymbol{n}}$. We denote by $\#\{\boldsymbol{F}\}$ the cardinality of $\boldsymbol{F}$. A convex polytope is the convex hull of a finite subset of $\boldsymbol{R}^{n}$. A lattice polytope is a polytope with its vertices in $\boldsymbol{Z}^{\boldsymbol{n}}$. A couple of years ago, R.J.Gardner, P.Gronchi and C.Zong proposed the following discrete version of the Aleksandrov problem:

If $\boldsymbol{C}_{1}$ and $\boldsymbol{C}_{2}$ are two origin-symmetric convex lattice polytopes such that $\#\left\{\boldsymbol{C}_{1} \mid \boldsymbol{u}^{\perp}\right\}=\#\left\{\boldsymbol{C}_{2} \mid \boldsymbol{u}^{\perp}\right\}$ for every $\boldsymbol{u} \in \boldsymbol{S}^{\boldsymbol{n}-1}$, do $\boldsymbol{C}_{1}$ and $\boldsymbol{C}_{2}$ coincide up to a translation?

Remark 1 In [1], Gardner, Gronchi and Zong discovered a counterexample to this problem in $\boldsymbol{Z}^{2}$ with cardinality 11(Figure 1).
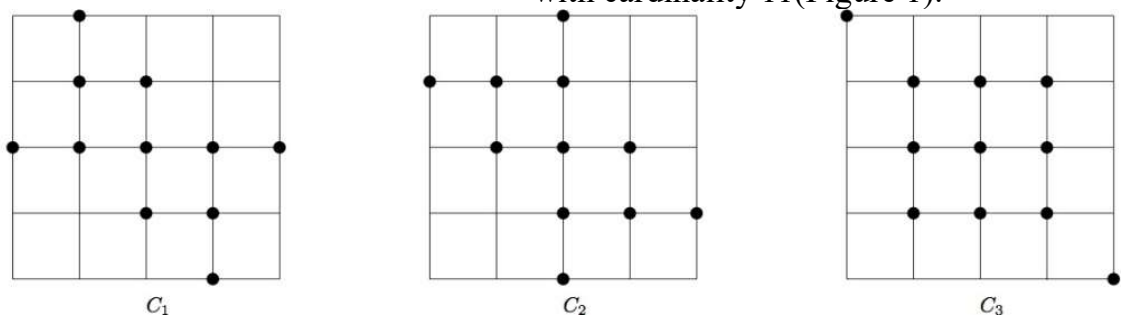

Figure 1: Counterexample in $\boldsymbol{Z}^{2}$ with cardinality 11 
In [7], Zhou proved that the counterexample discovered by Gardner, Gronchi and Zong was the only counterexample in $\boldsymbol{Z}^{2}$, up to unimodular transformations and with cardinality not larger than 17 . In [5], Xiong proved that an origin symmetric convex lattice set can be uniquely determined by its lattice projection counts if $\boldsymbol{y}$-coordinate's absolute value is not bigger than 2 for every point in this origin symmetric convex lattice set and its cardinality is not 11 .

Considering that in the discrete Aleksandrov's projection problem, counting of projection of a convex lattice set is important. In [3], Gu and Si presented an algorithm for counting of projection of a convex lattice set based on the coordinate matrix and its properties.

Gardner, Gronchi and Zong also asked in [1]: under what additional condition the discrete version of the Aleksandrov problem has an affirmative answer in $\boldsymbol{Z}^{2}$ ?

As one answer for the above problem, in [6], Zhang proved the following result.

Theorem 2 (Zhang) Let $\boldsymbol{C}_{1}$ and $\boldsymbol{C}_{2}$ be originsymmetric convex lattice sets in $\boldsymbol{Z}^{2}$. If

$$
\#\left\{\boldsymbol{C}_{1} \mid \boldsymbol{u}^{\perp}\right\}=\#\left\{\boldsymbol{C}_{2} \mid \boldsymbol{u}^{\perp}\right\}
$$

and

$$
\#\left\{2 \boldsymbol{C}_{1} \mid \boldsymbol{u}^{\perp}\right\}=\#\left\{2 \boldsymbol{C}_{2} \mid \boldsymbol{u}^{\perp}\right\}
$$

for all $\boldsymbol{u} \in \boldsymbol{Z}^{2}$, then $\boldsymbol{C}_{1}=\boldsymbol{C}_{2}$.

For a convex lattice set $\boldsymbol{C}$, the discrete perimeter $\#\left\{\partial\left(\boldsymbol{C} \mid \boldsymbol{u}^{\perp}\right)\right\}$ is defined by

$$
\#\left\{\partial\left(C \mid u^{\perp}\right)\right\}=\#\left\{\left(C \mid u^{\perp}\right) \cap \partial\left(\operatorname{conv}(C) \mid u^{\perp}\right)\right\} .
$$

In [4], Ryabogin, Yaskin and Zhang introduced the discrete perimeter as substitute for

$$
\#\left\{\boldsymbol{C}_{1} \mid \boldsymbol{u}^{\perp}\right\}=\#\left\{\boldsymbol{C}_{2} \mid \boldsymbol{u}^{\perp}\right\} \text { for every } \boldsymbol{u} \in \boldsymbol{S}^{\boldsymbol{n}-1},
$$

in the discrete version of the Aleksandrov problem. And they got the following theorem.

Theorem 3 (Ryabogin-Yaskin-Zhang) Let $\boldsymbol{C}_{1}$ and $\boldsymbol{C}_{2}$ be origin-symmetric convex lattice sets in $\boldsymbol{Z}^{3}$ which has non-empty interior. If the discrete perimeters of $\boldsymbol{C}_{1} \mid \boldsymbol{u}^{\perp}$ and $\boldsymbol{C}_{2} \mid \boldsymbol{u}^{\perp}$ are equal for all $\boldsymbol{u} \in \boldsymbol{Z}^{3}$, then $\boldsymbol{C}_{1}=\boldsymbol{C}_{2}$.

In higher dimensions, Ryabogin, Yaskin and Zhang also obtained an analogous result when $\operatorname{conv}\left(\boldsymbol{C}_{1}\right)$ and $\operatorname{conv}\left(\boldsymbol{C}_{2}\right)$ are zonotopes.

Let $\boldsymbol{C}\left(\boldsymbol{Z}^{2}\right)$ denote the set of all origin-symmetric convex lattice sets in $\boldsymbol{Z}^{2}$. For $\boldsymbol{C}_{1}, \boldsymbol{C}_{2}, \boldsymbol{C}_{3}$ in Figure 1, denote the set $C\left(Z^{2}\right) \backslash\left\{C_{1}, C_{2}, C_{3}\right\}$ by $C^{*}\left(Z^{2}\right)$.

Denote by $\boldsymbol{G L}_{2}(\boldsymbol{R})$ the general linear group in $\boldsymbol{R}^{2}$. Suppose that the discrete version of the Aleksandrov theorem is true in $\boldsymbol{C}^{*}\left(\boldsymbol{Z}^{2}\right)$, then we have the following theorem.
Theorem 4 If $C_{1}, C_{2} \in C^{*}\left(Z^{2}\right) \quad$ and

$\boldsymbol{g} \in \boldsymbol{G} \boldsymbol{L}_{2}(\boldsymbol{R})$ such that

$\#\left\{g C_{1} \mid g u^{\perp}\right\}=\#\left\{g C_{2} \mid g u^{\perp}\right\}$ for every $u \in S^{1}$, then $\boldsymbol{C}_{1}$ and $\boldsymbol{C}_{2}$ coincide up to a translation.

Proof:

Since $\boldsymbol{g} \in \boldsymbol{G} \boldsymbol{L}_{2}(\boldsymbol{R})$, the determinant of $\boldsymbol{g}$ is not zero, i.e. $\boldsymbol{g}$ is reversible.

Thus $\boldsymbol{R}^{2}$ is mapped to $\boldsymbol{g} \boldsymbol{R}^{2}$, i.e $\boldsymbol{R}^{2}$. But note that the lattice $\boldsymbol{Z}^{2}$ be replaced by $\boldsymbol{g} \boldsymbol{Z}^{2}$.

By $\#\left\{\boldsymbol{g} \boldsymbol{C}_{1} \mid \boldsymbol{g} \boldsymbol{u}^{\perp}\right\}=\#\left\{\boldsymbol{g} \boldsymbol{C}_{2} \mid \boldsymbol{g} \boldsymbol{u}^{\perp}\right\}$ and the reversibility of $\boldsymbol{g}$, we have

$\#\left\{\boldsymbol{C}_{1} \mid \boldsymbol{u}^{\perp}\right\}=\#\left\{\boldsymbol{C}_{2} \mid \boldsymbol{u}^{\perp}\right\} \quad$ for $\quad \boldsymbol{C}_{1}, \boldsymbol{C}_{2} \in \boldsymbol{C}^{*}\left(\boldsymbol{Z}^{2}\right)$ and $\boldsymbol{u} \in \boldsymbol{S}^{1}$.

By the hypothesis, the discrete version of the Aleksandrov theorem is true in $C^{*}\left(Z^{2}\right)$, we have $C_{1}$ and $\boldsymbol{C}_{2}$ coincide up to a translation.

Therefore, we can get the main result of the theorem.

\section{Conjectures about the convex lattice set}

All the results above show that the discrete Aleksandrov's projection problem need additional hypotheses. Motivated by the work of Gardner, Gronchi, Zong, Ryabogin, Yaskin and Zhang, some conjections about the projection of the convex lattice set are given.

In graph theory, Kuratowski's theorem states some characterization of planar graphs. A finite graph is planar if and only if it does not contain a subgraph that is a subdivision of $\boldsymbol{K}_{5}$ (the complete graph on five vertices) or of $\boldsymbol{K}_{3,3}$ (complete bipartite graph on six vertices).

Theorem 5 (Kuratowski) If a graph $\boldsymbol{G}$ is non-planar, then it must contain a subdivision of $\boldsymbol{K}_{3,3}$ or $\boldsymbol{K}_{5}$ (or both).

Let $\boldsymbol{C}\left(\boldsymbol{Z}^{n}\right)$ denote the set of all origin-symmetric convex lattice sets in $\boldsymbol{Z}^{\boldsymbol{n}}$. Considering the Kuratowski's theorem, for the discrete Aleksandrov's projection problem, the following revised conjectures are given.

Conjecture 1 Let $\boldsymbol{C}_{1}, \boldsymbol{C}_{2}$ belong to some subset of $\boldsymbol{C}\left(\boldsymbol{Z}^{n}\right)$ and

$$
\#\left\{\boldsymbol{C}_{1} \mid \boldsymbol{u}^{\perp}\right\}=\#\left\{\boldsymbol{C}_{2} \mid \boldsymbol{u}^{\perp}\right\}
$$

for every $\boldsymbol{u} \in \boldsymbol{S}^{\boldsymbol{n}-1}$. Then $\boldsymbol{C}_{1}$ and $\boldsymbol{C}_{2}$ coincide up to a translation.

The similar question is about the intersection of convex lattice polytope. 
Conjecture 2 Let $\boldsymbol{C}_{1}, \boldsymbol{C}_{2}$ belong to some subset of $\boldsymbol{C}\left(\boldsymbol{Z}^{n}\right)$ and

$$
\#\left\{\boldsymbol{C}_{1} \cap \boldsymbol{u}^{\perp}\right\}=\#\left\{\boldsymbol{C}_{2} \cap \boldsymbol{u}^{\perp}\right\}
$$

for every $\boldsymbol{u} \in \boldsymbol{S}^{\boldsymbol{n}-1}$. Then $\boldsymbol{C}_{1}$ and $\boldsymbol{C}_{2}$ coincide up to a translation.

Remark 2 For the above two conjectures, the some subset may not exist. The discovery of further relationship between discrete and continuous should be the key for finding these subsets in $\boldsymbol{C}\left(\boldsymbol{Z}^{\boldsymbol{n}}\right)$.

The Busemann-Petty problem[2], asks the following question.

Suppose that $\boldsymbol{C}_{1}$ and $\boldsymbol{C}_{2}$ are origin-symmetric convex bodies in $\boldsymbol{R}^{\boldsymbol{n}}$ such that

$$
v_{n-1}\left(C_{1} \cap u^{\perp}\right) \leq v_{n-1}\left(C_{2} \cap u^{\perp}\right)
$$

does it follow that $\boldsymbol{v}_{n}\left(C_{1}\right) \leq \boldsymbol{v}_{n}\left(C_{2}\right)$ ?

This famous problem has been solved by series of papers from many authors. An analogy question in $\boldsymbol{Z}^{\boldsymbol{n}}$ is as follows.

Conjecture 3 Let $\boldsymbol{C}_{1}, \boldsymbol{C}_{2}$ belong to some subset of $\boldsymbol{C}\left(\boldsymbol{Z}^{\boldsymbol{n}}\right)$. Suppose that $\#\left\{\boldsymbol{C}_{1} \mid \boldsymbol{u}^{\perp}\right\} \leq \#\left\{\boldsymbol{C}_{2} \mid \boldsymbol{u}^{\perp}\right\}$ for every $\boldsymbol{u} \in \boldsymbol{S}^{\boldsymbol{n}-1}$, is $\boldsymbol{C}_{1}$ included in $\boldsymbol{C}_{2}$ by some suitable translation?

\section{ACKNOWLEDGEMENTS}

This study is supported by the Fundamental Research Funds for the Central Universities (2017ZY44,2015ZCQLY-01), the State Forestry Administration 948 Project(2013-4-66)

\section{References}

1. R.Gardner, P.Gronchi and C.Zong, Sums, Projections, and Sections of Lattice Sets, and the Discrete Covariogram, Discrete Comput. Geom. 34 (2005) 391-409.

2. P.M.Gruber, Convex and Discrete Geometry, Springer-Verlag, 2007.

3. Y.Gu and L.Si, The Projections of Convex Lattice Sets of Points in E2, Mathematical Problems in Engineering, vol. 2016, Article ID 7351861, 2016. doi:10.1155/2016/7351861.

4. D.Ryabogin, V.Yaskin, N.Zhang, Unique Determination of Convex Lattice Sets, Discrete Comput Geom 57(2017)582-589.

5. H. Xiong, On a discrete version of Alexandrov's projection theorem, Acta Mathematica Sinica (English Series)29(2013)1597-1606.

6. N.Zhang, An analogue of the Aleksandrov projection theorem for convex lattice polygons, Proc. Amer. Math. Soc.145(2017)2305-2310.

7. J.Zhou, On the Projections of Convex Lattice Sets, Acta Mathematica Sinica (English Series) 26 (2010) 1969-1980. 